\title{
BMJ Open Association between diabetes-related self-care activities and positive health: a cross-sectional study
}

\author{
Fang-Fang Zhao, ${ }^{1,2}$ Riitta Suhonen, ${ }^{1,3}$ Jouko Katajisto, ${ }^{4}$ Minna Stolt, ${ }^{\oplus 1}$ \\ Helena Leino-Kilpi ${ }^{1,5}$
}

To cite: Zhao F-F, Suhonen $R$, Katajisto J, et al. Association between diabetes-related self-care activities and positive health: a crosssectional study. BMJ Open 2019;9:e023878. doi:10.1136/ bmjopen-2018-023878

- Prepublication history and additional material for this paper are available online. To view these files, please visit the journal online (http://dx.doi. org/10.1136/bmjopen-2018023878).

Received 3 May 2018

Revised 24 March 2019

Accepted 28 May 2019

D Check for updates

(c) Author(s) (or their employer(s)) 2019. Re-use permitted under CC BY-NC. No commercial re-use. See rights and permissions. Published by BMJ.

${ }^{1}$ Department of Nursing Science, Faculty of Medicine, University of Turku, Turku, Finland

${ }^{2}$ Department of Nursing Science, Faculty of Medicine, Nantong University, Nantong, China

${ }^{3}$ Turku University Hospital and City of Turku, Welfare Division, Turku, Finland

${ }^{4}$ Department of Mathematics and Statistics, University of

Turku, Turku, Finland

${ }^{5}$ Turku University Hospital, Turku, Finland

Correspondence to

Fang-Fang Zhao;

fangfang.zhao@utu.fi

\section{ABSTRACT}

Objectives The study aimed to examine the diabetesrelated self-care activities (DRSCA) in association with positive health consisting of resilience, subjective wellbeing (SWB) and disease-related quality of life (DRQoL), and the associations of background information with resilience, SWB and DRQoL.

Design A cross-sectional study.

Methods A convenience sample of people with type 2 diabetes (T2DM) was recruited from two tertiary hospitals in eastern China. Self-reported survey data included DRSCA, resilience, SWB, DRQoL, and background information comprising demographics and social support. Data were analysed using hierarchical multiple regression analyses.

Results Two hundred and forty-six valid questionnaires were analysed. Among the components of positive health, hierarchical multiple regression analysis showed that DRSCA were significantly associated with improved SWB $(\beta=0.140, p=0.020)$, but not with resilience and DRQoL after adjusting for background information. Participants with higher level of education had higher resilience and men had higher SWB than women. Social support $(\beta=0.186, p=0.003)$ in Model 1 and resilience $(\beta=0.298$, $\mathrm{p}<0.001$ ) in Model 2 were positively associated with SWB. Conclusions This study indicated an association between DRSCA and one component of positive health, namely SWB. This finding could help motivate patients to perform DRSCA and to improve SWB in people with T2DM. In addition, the fact that demographics, for example, gender, were associated with SWB and education was associated with resilience also needs to be accommodated when aiming to improve SWB and build resilience.

Trial registration number NCT02594748

\section{INTRODUCTION}

Diabetes is a chronic disease and its self-management is complex, time-consuming and life-long, posing a challenge throughout life. ${ }^{12}$ Diabetes has increasingly become a public health problem worldwide. More than 425 million people were living with diabetes in 2017 and people with diabetes are predicted to increase to 693 million by $2045 .^{3}$ In China, the prevalence rate of diagnosed diabetes was estimated to be about $9.7 \%$ among people

\section{Strengths and limitations of this study}

This study provided information about the association of diabetes-related self-care activities (DRSCA) with positive health consisting of resilience, subjective well-being (SWB) and disease-related quality of life (DRQoL) and may help encourage the performance of DRSCA and draw attention to improving positive health among people with type 2 diabetes.

- When analysing the association of DRSCA with resilience, SWB and DRQoL, patients' background information was used as a covariate, which made the evaluation more specific.

- Causal association between DRSCA and resilience, SWB and DRQoL cannot be determined because of the cross-sectional design.

- The non-probability sampling method limits the generalisability of the results.

aged $20-79$ years in $2017 .{ }^{3}$ Diabetes can cause long-term complications and also imposes an economic burden on the individuals. In mainland China, there are mainly four types of medical insurance; on average, slightly more than $30 \%$ of the total cost of the treatment was paid out-of-pocket by the patients. ${ }^{4}$

Preventing complication of diabetes is an urgent need. Diabetes management can prevent disease complication and diabetes-related self-care activities (DRSCA) are the key component of overall diabetes management. DRSCA refer to an active, effective disease management strategy, self-organisation of one's own life in order to control the disease, mainly including, but not limited to, medication adherence, diet control, blood sugar monitoring, physical activities and foot care. ${ }^{5}$ These five domains that DRSCA cover are essential for self-management. Self-report measuring the domains of DRSCA has been the most commonly used method in research and has proven to be appealing to researchers and clinicians alike. ${ }^{6}$

Successful diabetes management with sufficient DRSCA can control disease progression ${ }^{7}$ 
and it is thus very important to improve DRSCA. However, it is often difficult for people to engage daily in the selfcare activities needed for good glycaemic control. ${ }^{8} \mathrm{~A}$ cross-sectional study showed that only $13.6 \%$ of patients achieved the goal for Haemoglobin A1c level lower than $6.5 \%$ which measures glycaemic control. ${ }^{9}$ The overall level of self-care activities index was about 50\%-60\% in previous studies. ${ }^{11}$ The least frequently reported DRSCA was self-monitoring of blood glucose, which may be due to the cost or fear of pain, ${ }^{11}$ even though self-monitoring of blood glucose is important for monitoring disease control. ${ }^{5}$

The importance of DRSCA for patients has been noted by a number of researchers ${ }^{12} 13$ and the promoting factors of DRSCA have been studied from different perspectives. Encouraging people with type 2 diabetes (T2DM) to improve their DRSCA levels by providing supportive information has gained increasing attention. ${ }^{4} 15$

To encourage patients to be responsible for their performance of DRSCA, additional information is needed about the influence of DRSCA on positive health. Previously, a qualitative meta-synthesis showed that patients' perceptions of the positive consequences of DRSCA influenced their self-care efforts. ${ }^{16}$ In addition, a systematic review indicated that perceived benefits, belonging to the components of the Health Belief Model, ${ }^{17}$ were associated with higher level of DRSCA. ${ }^{18}$ Therefore, identifying the association of DRSCA with positive health may explain more health benefits of DRSCA and may promote patients to assume more responsibility for their daily DRSCA.

On the other hand, there is also a need to identify plausible relationships between DRSCA and positive health from a general perspective. Epidemiological work and public health guidelines focus on preventing and restoring negative functioning while there is less attention on promoting positive health. ${ }^{19}$ Poor health behaviours have been shown to be associated with increased mortality or morbidity. ${ }^{20}$ However, far less is known about the health behaviours associated with positive health. ${ }^{19}$ There is a need to examine whether health behaviours such as DRSCA are associated with positive health. Identifying the association of DRSCA with positive health in our study would provide a useful step in guiding future diabetes care research to include positive health as an outcome indicator. It may also provide a basis for further research aimed at improving positive health of people with T2DM to consider including the role of the DRSCA.

Seeman ${ }^{21}$ developed a model of positive health, referring to positive health as effective human functioning and effective coping and response. However, the definition of positive health is ambiguous and there is no instrument for measuring it. ${ }^{22}$ For example, Locker and Gibson suggested the definition of positive health as covering a broad range of constructs such as well-being, coping and adaptation. ${ }^{22}$ Cloninger $e t a l^{23}$ summarised that positive health comprises wellness and healthy functioning. In addition, Seligman ${ }^{24}$ suggested that the global notion of positive health can be broken down into three kinds of independent variables: subjective (a sense of physical well-being, hardiness, confidence and optimism), biological and functional. Therefore, based on the common meaning of positive health from the previous definitions, we used resilience, subjective well-being (SWB) and disease-related quality of life (DRQoL) as components of positive health. Resilience represents coping, adaptation, recovery from hardships and optimistic thinking; SWB represents satisfaction of physical and mental well-being and DRQoL represents physical and social function. In this study, DRSCA are hypothesised to be positively associated with positive health consisting of resilience, SWB and DRQOL, which are discussed below in separate paragraphs.

Resilience is the coping and adapting capacity to recover from impaired function after a challenge or adversity, which helps one to stay optimistic. ${ }^{2526}$ There has been a growing interest in resilience as it has been shown to protect against psychiatric illness. ${ }^{27}$ The prevalence of such illnesses is high among patients with diabetes. ${ }^{28}$ Moreover, resilience has been regarded as an essential factor contributing to longevity and well-being. ${ }^{29}$ Resilience is not necessarily an intrinsic attribute. Resilience theorists believe that resilience can be learnt, developed and enhanced through an individual's lived experience and activities. ${ }^{30} 31 \mathrm{~A}$ previous empirical study indicated that healthy self-care strategies can promote resilience among veterinary staff. ${ }^{32}$ Better DRSCA teach people to adopt multiple skills for more self-disciplined lives, which may be helpful in building resilience. However, this association has not been tested in earlier studies.

SWB is the integration of a person's satisfaction of physical, mental health and positive emotions to foster health. ${ }^{33}$ High levels of SWB have been found to be beneficial to good health, longevity and optimal human functioning, which has become an important target that practitioners aim to achieve. ${ }^{34}$ Therefore, SWB has been emphasised as an essential target in health promotion programmes. ${ }^{35} 36$ Self-care strategy, such as physical activities, has been indicated to improve or maintain SWB in Swedish adults. ${ }^{37}$ Based on the very scarce published data, ${ }^{37}$ DRSCA was hypothesised to be associated with improved SWB among people with T2DM in this study.

DRQoL has been used to evaluate how diabetes impacts physical and social functions in patients' life domains and the perceived importance of these life domains for them. ${ }^{38}$ Although previous studies have supported the notion that better DRSCA leads to increased health-related quality of life ${ }^{39} 40$ to our knowledge, there are no studies about the direct association between DRSCA and DRQoL among people with T2DM conducted in the eastern region of mainland China. Understanding their association in different areas may provide additional information for diabetes care.

The objectives of this study are:

1. To explore whether an association exists between DRSCA and positive health that consists of resilience, 
SWB and DRQoL, which provides the premise for their causative relationships before entering into intervention study.

2. To explore how background information is associated with positive health that consists of resilience, SWB and DRQoL. Family history of diabetes and whether the patient experienced symptoms before the diabetes diagnosis were also added to the background information. The reasons for including them were as follows: Family history is an independent risk factor for chronic diseases includingT2DM. ${ }^{41}$ Patients felt that family members' knowledge of diabetes helped to provide diabetes-specific support. ${ }^{42}$ As for the presence of symptoms before the diabetes diagnosis, we considered that if the person had no symptoms prior to diagnosis on physical examination, he/she may feel that the onset of disease was sudden and there was no psychological preparation. In this case, the adaptation process may be longer compared with those who experienced symptoms before being diagnosed. An inadequate and prolonged adaptive process may cause lower well-being and anxiety. ${ }^{43}$

This study may provide helpful information both for encouraging patients' DRSCA and for improving resilience, SWB and DRQoL in health promotion programmes.

\section{METHODS}

\section{Design and participants}

A descriptive, cross-sectional study was conducted. The data were collected using convenience sampling among patients with T2DM seeking medical care at two university-affiliated hospitals in Nantong city, Jiangsu Province, located in eastern China between summer 2015 and summer 2016. The following inclusion criteria used in the recruitment of the study sample were reported ${ }^{44}$ : age $18+$ years; duration of T2DM for at least 3 months; no serious complications or cognition disorders and willing to participate and provide written informed consent. The exclusion criteria were as follows: refusal to participate and serious complications.

\section{Instruments}

The instruments used in this study were self-reported measures. This study surveyed what patients had done in their self-care: patients were asked to recall their DRSCA over the past week at home to lessen reactivity where people may modify their behaviour when they know they are being observed. ${ }^{45}$ Equipment for objective measurement of self-care activities has not been in universal use among Chinese patients, and the concept of positive health in the study involved subjective sense and perceptions (eg, positive emotion; perception of the impact of diabetes on daily life); therefore, self-reported measures were used. The instruments included background information, DRSCA and components for positive health comprising resilience, SWB and DRQoL.
The supplemented information about the instruments is shown online supplementary file (Appendices-DRSCA and positive health).

\section{Background information}

Background information included demographics and social support. Demographics included age, gender, education, duration of diabetes, whether the patient had received standardised diabetes education, living alone, family history of diabetes and whether the patient had experienced symptoms before diabetes diagnosis, perceived complications (Yes/No), smoking (Yes/No), drinking alcohol (Yes/No) as well as clinical characteristics including systolic blood pressure (SBP), diastolic blood pressure (DBP), height (in $\mathrm{cm}$ ), weight (in $\mathrm{kg}$ ). Body mass index (BMI) was calculated as weight/ height ${ }^{2}\left(\mathrm{~kg} / \mathrm{m}^{2}\right)$.

Social support was measured by the Social Support Rating Scale. ${ }^{46}$ It has 10 items and 3 dimensions: subjective support, objective support and support utilisation. Response option of eight items was from 1 to 4 . One item among them has five subquestions and each question was scored from 1 to 4 . Two items were scored 0-9 based on the support resources. The Cronbach's $\alpha$ was 0.70 in the present sample. Social support scores $\leq 44$ are classified as low support level while those $>44$ are classified as high support level. ${ }^{47}$

\section{Diabetes-related self-care activities}

DRSCA were measured by the Diabetes Self-Management Scale developed by Yin $e t a t^{8}$ which is based on core items of the widely used scale of Summary of Diabetes DRSCA. ${ }^{5}$ The scale consists of eight items and five dimensions including regulating diet, physical activities, medications, blood sugar monitoring and foot care. The participants described on how many days in the past week they had carried out DRSCA following doctor's advice. The items stated: On how many days have you followed the prescription of medication; regulated your diet for diabetes; done physical activities for $30 \mathrm{~min}$ or more; exercised (except for homework and work), for example, walking, Tai chi, swimming, jogging or bicycling; tested your blood sugar; tested your blood sugar the times recommended by doctor; checked your feet and dried between your toes after washing in the past week? Items were rated on an 8-point Likert scale, a higher score indicating a better level of self-care. The scale Cronbach's a was $0.68 .^{48}$

\section{Components of positive health}

\section{Resilience}

Resilience was measured by the 10-item Connor-Davidson Resilience Scale (10-item CD-RISC), modified by Campbell-Sills and Stein ${ }^{49}$ from the original scale developed by Connor and Davidson. ${ }^{25}$ The 10 -item resilience scale is a self-administered questionnaire to measure resilience ability, with five Likert-type response options from 0 (never) to 4 (almost always). It has a single dimension and a total score range between 0 and 40 , a higher score 
representing a higher level of resilience. Cronbach's $\alpha$ was 0.94 in this study.

\section{Subjective well-being}

SWB was evaluated with the WHO-5 Well-Being Index developed from the WHO-10 Well-Being Index. ${ }^{5051}$ It is a unidimensional scale consisting of five positively worded items scored on a 6-point Likert scale ranging from 0 (not present) to 5 (constantly present). The total raw scores range from 0 to 25 . The raw scores are then converted to 0-100 multiplied by 4 , from worst thinkable well-being to best thinkable well-being. As a short and generic global rating scale, the WHO-5 Well-Being Index is one of the most widely used instruments measuring SWB covering basic life perceptions of well-being. ${ }^{52}$ In this study, the internal consistency measured with Cronbach's $\alpha$ was 0.84 .

\section{Disease-related quality of life}

DRQoL was measured by the ADDQoL-19 (Audit of Disease-related quality of life), which is designed to assess how individuals perceive the impact of DM on 19 pairs of items representing life domains. ${ }^{1}$ For each of these items, respondents first select the impact of diabetes on the applicable domain from -3 (most negative impact) to +1 (positive impact) and then rank the importance of the domain for their QoL from 3 (very important) to 0 (not at all important). A weighted impact score for each domain is given by multiplying impact scores and corresponding important scores, ranging from -9 (most negative impact) to +3 (most positive impact). The average weighted impact (AWI) score is calculated by summarising the weighted impact scores and dividing the sum by the number of the domains. The AWI ranges from 3 (positive impact) to -9 (most negative impact). ${ }^{1}$ The Chinese version of the ADDQoL-19 has a Cronbach's $\alpha$ value of $0.94 .^{53}$ In this study, the internal consistency measured with Cronbach's $\alpha$ was 0.9 .

\section{Ethical considerations}

This study adhered to the ethical principles of the Helsinki Declaration and was approved by the Ethical Committee of a University affiliated Hospital (Code: 2015-120). Permission for the study was given by the participating hospitals. Numbers were assigned to participants to ensure anonymity and information was kept confidential. Written informed consent was obtained from participants. The study scales were used with permission by the original authors or authorised coordinator (WHO-5 WellBeing Index) received via email when usage permission was required.

\section{Data collection procedures}

Recruitment was conducted at two University affiliated hospitals of Nantong University. The participants who met the inclusion criteria after screening were recruited shortly after being admitted to hospital. The participants in this study came to hospital for help mainly for adjusting their medication treatment including oral antidiabetic drugs (OADs) alone or OADs in combination with insulin and/or glucagon-like peptide-1 receptor agonist, or using insulin alone, to control glycaemic level and to prevent or treat complication. The potential participants were approached and informed about the purpose, meaning, content and procedures of the study and were asked to sign an informed consent if they agreed to participate. Participation was totally voluntary and the participants were told they could withdraw their participation at any time.

Before data collection, the research assistants were trained by the main researcher to perform the data collection procedure consistently. The training included information about the study, ethical issues, interviewing techniques and questionnaire coding. The main researcher also demonstrated the collection procedure and observed the process of data collection when it was performed for the first time, making corrections if necessary. The data were collected using structured questionnaires. Questions were read out loud to participants with poor vision, for example. For ethical consideration, the anonymity of the data analysis was explained. Each patient was assigned a unique code which was used in the analysis. The required sample size was calculated in $\mathrm{G}^{*}$ power statistical software by setting the alpha at 0.05 , the effect size at 0.10 , the power at 0.90 ; the number of tested and total predicted factors was set at 15 . The sample size for multiple regression analysis was calculated as 249 (fixed model, $\mathrm{R}^{2}$ increase).$^{54}$ The required sample size was about 300 to allow for $20 \%$ invalid response rate. Of the patients who were approached to invite them to participate in the study, 310 (about 70\%) took part in the study.

\section{Data analysis}

IBM SPSS 23.0 was used to analyse the data. Before analysis, errors such as data transfer errors and extreme outliers of variables in this study were removed. ${ }^{55} \mathrm{An}$ extreme outlier was less than first quartile Q1 $-3 * I Q R$ or greater than $Q 3+3 * I Q R$. Sensitivity analyses including and excluding these outliers were conducted separately, and the same general conclusion was drawn, except that gender was not significant for resilience in Model 1 in the hierarchical multiple regression analysis when the outliers were included. The general results are not sensitive to the outliers and are robust. Missing values of study variables accounted for no more than $5 \%$ except for BMI, SBP and DBP (12\%) and were replaced by the Values-Expectation-Maximisation method. Descriptive statistics of frequency, percentage, mean and SD were calculated to express data. Internal consistency reliability was computed for the scales. Pearson's correlation was used to explore the correlation between DRSCA, resilience, SWB and DRQoL as well as the correlation between SBP, DBP, BMI with resilience, SWB and DRQoL. Hierarchical multiple regression analysis was conducted to explore the associations of DRSCA with positive health while adjusting for covariates (background information, see table 1) based 
Table 1 Association of background information with components of positive health $(n=246)$

\section{Positive health}

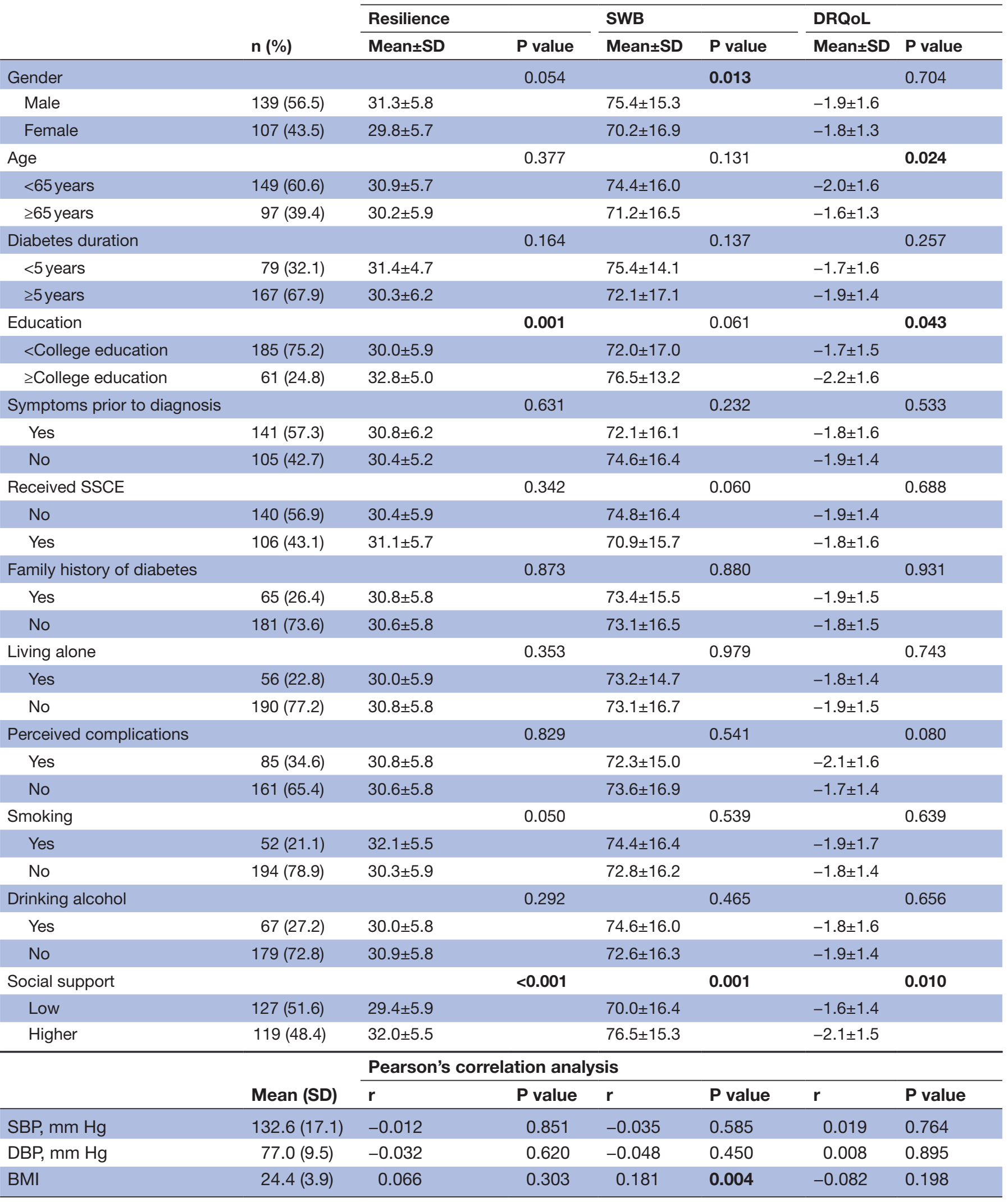

Bold $p$ values were less than 0.05 .

BMI, body mass index; DBP, diastolic blood pressure; DRQoL, disease-related quality of life; SBP, systolic blood pressure; SSCE, standardised self-care education; SWB, subjective well-being . 


\begin{tabular}{|c|c|c|c|c|}
\hline & \multicolumn{4}{|c|}{ Positive health } \\
\hline & DRSCA & Resilience & SWB & DRQoL \\
\hline \multicolumn{5}{|l|}{ DRSCA } \\
\hline$r$ & 1 & & & \\
\hline$P$ value & & & . & \\
\hline \multicolumn{5}{|l|}{ Resilience } \\
\hline$r$ & $0.165^{\star}$ & 1 & & \\
\hline $\mathrm{P}$ value & 0.009 & & & \\
\hline \multicolumn{5}{|l|}{ SWB } \\
\hline$r$ & $0.205^{\star}$ & $0.346^{\star}$ & 1 & \\
\hline$P$ value & 0.001 & 0.000 & & \\
\hline \multicolumn{5}{|l|}{ DRQoL } \\
\hline$r$ & -0.115 & -0.091 & -0.100 & 1 \\
\hline $\mathrm{P}$ value & 0.072 & 0.154 & 0.118 & \\
\hline
\end{tabular}

${ }^{*}$ Correlation is significant at the 0.01 level (2-tailed). DRQoL, disease-related quality of life; DRSCA, diabetes-related self-care activities; SWB, subjective well-being.

on the results from t-test and Pearson's correlation analysis (see table 2). $\mathrm{P}<0.05$ indicated statistical significance.

\section{Patient and public involvement (PPI) statement}

Patients were not involved in research activities including designing and conducting the study.

\section{RESULTS}

\section{Characteristics of the sample}

Two hundred and forty-nine valid questionnaires were included in this study after excluding incomplete questionnaires and unusable ones reporting hospital care as 1 week's DRSCA. After excluding extreme outliers of variables in this study, the data set for analysis comprised 246 valid questionnaires. The average age of these participants was 59.6 years $(\mathrm{SD}=13.4$, range 22-85). Although the incidence of T2DM is on the rise among young people ${ }^{56}$ typically T2DM starts in middle or older age. ${ }^{57}$ Therefore, age 65 was chosen as an age cut-off. Older adults ( $\geq 65$ years) accounted for $39.4 \%$ of the participants in this study. Slightly less than half $(43.5 \%)$ of the participants were women. Diabetes duration was on average 9.9 years, ranging from 0.5 to 37 years. Slightly more than half $(57.3 \%)$ of the participants had experienced symptoms prior to the diagnosis, and about $43.1 \%$ had received standardised self-care education in the past. The majority $(75.2 \%)$ of the participants had less education than a college degree. About one-fourth of the participants had a family history of diabetes $(26.4 \%)$. About a fourth (22.8\%) of those living alone and $48.4 \%$ of all participants self-reported that they had high support (score $>44$ ) (table 1).
Level of DRSCA and components of positive health

The scores of self-reported DRSCA, resilience, SWB and DRQoL were at moderate level. DRSCA had a mean score of 34.9 ( $\mathrm{SD}=11.4$, score index using actual score divided by possible maximum score, $62 \%$ ). Resilience had a mean score of $30.7(\mathrm{SD}=5.8$. score index, $77 \%)$. The mean score of SWB was 73.2 ( $\mathrm{SD}=16.2$, score index, $73 \%)$ and DRQoL was -1.9 with SD of 1.5 and possible score range of $3(-9)$.

\section{Association of background information with components of positive health}

The independent t-test results showed that men were more likely to have higher SWB compared with women. BMI was correlated with SWB. Participants aged $\geq 65$ years were more likely to have higher DRQoL. Participants with higher education seemed to have higher resilience but more impacted DRQoL. Participants with higher social support seemed to have higher resilience, SWB and perceived more impact in DRQoL.

\section{Correlations of DRSCA with components of positive health}

Based on correlation analysis, there were low but significant correlations (ranging from 0.165 to 0.346 ) between DRSCA, resilience and SWB (table 2).

\section{The association of DRSCA with components of positive health}

The association of DRSCA with positive health consisting of resilience, SWB and DRQoL was analysed after adjusting for covariates. Covariates were based on the statistically significant results from t-test and Pearson's correlation analysis (table 3 ).

For the dependent variable resilience, statistically significant background information from t-test including education and social support was entered in Step 1 (Model 1), SWB was entered in Step 2 (Model 2) and DRSCA in Step 3 (Model 3). The results showed that in Model 1, education $(\beta=0.182, p=0.004)$ and social support $(\beta=0.201, p=0.001)$ were significantly associated with resilience. In Model 2, SWB $(\beta=0.299, \mathrm{p}<0.001)$ was significantly associated with resilience. In Model 3, DRSCA ( $\beta=0.069, p=0.260)$ were not significant after adjusting for the covariates. The model explained $17.3 \%$ of the variance of resilience. Excluding the effects of demographics, social support and SWB, DRSCA had a non-significant relation with resilience.

For the dependent variable SWB, gender, BMI and social support were entered in Step 1 (Model 1), resilience in Step 2 (Model 2) and DRSCA in Step 3 (Model 3). In Model 1 , gender $(\beta=-0.134, p=0.033)$, BMI $(\beta=0.142, p=0.025)$ and social support $(\beta=0.186, p=0.003)$ were significantly associated factors of SWB. In Model 2, resilience $(\beta=0.298, p<0.001)$ was significantly associated with SWB, and in Model 3, DRSCA ( $\beta=0.140, p=0.020)$ were also significant. The model explained $18.6 \%$ of the variance of SWB. Excluding the effects of demographics, social support and resilience, DRSCA explained a significant part of the variance $(1.9 \%)$. 
Table 3 Hierarchical multiple regression analysis of the association between DRSCA and components of positive health

\begin{tabular}{|c|c|c|c|c|c|c|c|c|c|c|}
\hline \multirow{2}{*}{$\begin{array}{l}\text { DV } \\
\text { Positive health }\end{array}$} & \multirow[b]{2}{*}{ IV } & \multicolumn{3}{|c|}{ Model 1} & \multicolumn{3}{|c|}{ Model 2} & \multicolumn{3}{|c|}{ Model 3} \\
\hline & & B & $\beta$ & $P$ value & B & $\beta$ & P value & B & $\beta$ & $P$ value \\
\hline \multicolumn{11}{|l|}{ Resilience } \\
\hline & Education & 2.453 & 0.182 & 0.004 & 2.081 & 0.155 & 0.010 & 2.045 & 0.152 & 0.011 \\
\hline & SS & 2.340 & 0.201 & 0.001 & 1.683 & 0.145 & 0.017 & 1.562 & 0.134 & 0.028 \\
\hline & SWB & & & & 0.107 & 0.299 & $<0.001$ & 0.103 & 0.287 & $<0.001$ \\
\hline & DRSCA & & & & & & & 0.035 & 0.069 & 0.260 \\
\hline \multicolumn{11}{|l|}{ SWB } \\
\hline & Gender & -4.366 & -0.134 & 0.033 & -3.233 & -0.099 & 0.100 & -3.414 & -0.105 & 0.080 \\
\hline & BMI & 0.595 & 0.142 & 0.025 & 0.563 & 0.134 & 0.026 & 0.565 & 0.134 & 0.024 \\
\hline & SS & 6.034 & 0.186 & 0.003 & 3.878 & 0.120 & 0.050 & 3.141 & 0.097 & 0.113 \\
\hline & Resilience & & & & 0.832 & 0.298 & $<0.001$ & 0.780 & 0.280 & $<0.001$ \\
\hline & DRSCA & & & & & & & 0.199 & 0.140 & 0.020 \\
\hline \multicolumn{11}{|l|}{ DRQoL } \\
\hline & Age & 0.303 & 0.100 & 0.127 & 0.346 & 0.114 & 0.084 & & & \\
\hline & Education & -0.330 & -0.096 & 0.135 & -0.305 & -0.089 & 0.166 & & & \\
\hline & SS & -0.379 & -0.127 & 0.051 & -0.316 & -0.106 & 0.109 & & & \\
\hline & DRSCA & & & & -0.013 & -0.097 & 0.134 & & & \\
\hline
\end{tabular}

B, unstandardised coefficients; $\beta$, standardised coefficients; BMI, body mass index; DRQoL, disease-related quality of life; DRSCA, diabetesrelated self-care activities; DV, dependent variables; IV, independent variables; SS, social support.

For the dependent variable DRQoL, age, education and social support were entered in Step 1 (Model 1 ) and DRSCA in Step 2 (Model 2). In Model 1, age $(\beta=0.100, p=0.127)$, education $(\beta=-0.096, p=0.135)$ and social support $(\beta=-0.127, \mathrm{p}=0.051)$ were not significantly associated with DRQoL. In Model 2, DRSCA $(\beta=-0.097$, $\mathrm{p}=0.134)$ were also not significant. The model explained $5.6 \%$ of the variance of DRQoL. Excluding the effects of age, education and social support, DRSCA were not significantly associated with DRQoL.

These results showed that after adjusting for covariates, DRSCA were significantly associated with improved SWB, but not with resilience or DRQoL.

\section{DISCUSSION}

This study showed the level of DRSCA as well as resilience, SWB and DRQoL, which constitutes positive health. This study also explored the association of the components of positive health with DRSCA as well as with background information.

The score index of DRSCA was not high, supporting earlier findings. ${ }^{10}{ }^{12}$ However, we found a higher score index of DRSCA than that reported in another Chinese sample ${ }^{11}$ showing that the level of DRSCA is in general not optimistic. In the components of positive health, the score index of resilience was similar to a report from a previous study of people with chronic disease ${ }^{58}$ while the score of DRQoL was similar to previous studies conducted in Argentina ${ }^{59}$ and Australia. ${ }^{60}$ Although the score index of SWB in the study was moderate, it was still higher than among adolescents with type 1 diabetes. ${ }^{61}$ This indicates that further research aimed at enhancing SWB is needed for people with diabetes. These discrepancies might also be influenced by different measures, respondents' living arrangements and situation. The scores of all instruments were not high. The reason for this may be the situation of the participants who came to the hospital to get help to improve their health outcomes and reduce the risk of disease progression. We do not know for sure whether population-level DRSCA impacted the association between DRSCA and positive health. We judge the impact not to be substantial as the range of scores on the variables impacts the correlation ${ }^{62}$ and the study variables including DRSCA, resilience, SWB and DRQoL have some degree of variability (SD).

This study indicated that although DRSCA were positively correlated with resilience in Pearson correlation analysis, the association was non-significant after adjusting for background information. This finding indicates that compared with the association of social support and SWB with resilience, the association between DRSCA and resilience was weak in people with T2DM and different from a previous study conducted among veterinary staff. ${ }^{32}$ This implies that a high level of social support is more important than DRSCA in building resilience in people with T2DM. This study suggests that providing more social support resources can be a means of enhancing resilience.

DRSCA were not associated with increased DRQoL after adjusting for covariates. This finding showed that 
patients' life domains were impacted by diabetes and DRSCA did not increase or relieve the impact perceived by the patients. This result is different from an earlier study. ${ }^{39}$ In our study, the DRQOL was measured based on individuals' perceptions of the impact of DM on life domains and the importance of these domains for them. The discrepancy between the findings may be caused by the different perspective as compared with previous studies. The association between DRSCA and DRQoL reflected whether DRSCA influenced such perceptions and the result showed that the association between them was not significant. A possible explanation is that DRSCA are related to disease control, so they limit individuals from maintaining their original lifestyle and may therefore be considered demanding. As a result, patients may fail to feel that DRSCA lighten the impact of DM on some life domains in DRQOL, such as freedom to eat as they wish. One study ${ }^{1}$ found that if diabetes management and treatment permits dietary freedom and flexibility, it will lead to improved QoL for most people. The finding implied that patients' personal food preferences should be taken into account to create a tailor-made diet when drawing up a nutritional plan. ${ }^{63}$ Given that the variance of DRQoL accounted for by the model was not high, other factors such as the presence of mental health conditions should be considered ${ }^{2}$ in future studies.

Similar to a previous study in Swedish adults, ${ }^{37}$ the present analysis showed that DRSCA were positively associated with SWB. This finding strengthens the suggestion of the benefits of DRSCA in improving psychological health in chronic illnesses, ${ }^{64}$ which needs to be confirmed by studies using an intervention design. Although people still feel the impact of diabetes on life domains of DRQoL after the performance of DRSCA, they may experience positive mood and vitality in SWB due to achieving a disciplined lifestyle and self-care. From a public health perspective, SWB has been emphasised as an ultimate goal in health promotion programs, ${ }^{35} 36$ and recognising the association between DRSCA and SWB may contribute to both increased DRSCA and SWB in these programmes.

An interesting observation of our study is that people with higher education seemed to have higher resilience. Possible explanations include that people with higher education may have learnt more coping strategies, and coping strategies are linked to resilience. ${ }^{25}$ Men had higher SWB compared with women, which was in line with previous studies showing women to be more susceptible to emotional distress and emotional burden in their specific social roles than men. ${ }^{65}$ Contrary to what was expected, neither family history nor symptoms prior to diagnosis were associated with positive health. This insignificant association may be explained by the fact that the majority of patients in the study have had diabetes for over 5 years. During the process, they may to a certain extent have learnt how to manage the disease themselves. Individuals are considered to be adapting well when they act in a goal-oriented way during the adaptation process. ${ }^{66}$
In this study, the protective role of social support in resilience and SWB was observed among people with T2DM. Social support has been regarded as a positive factor contributing to mental health and promoting SWB, ${ }^{67}{ }^{68}$ which should be emphasised in supporting chronic care. ${ }^{68}$ In this study, resilience was also found to be associated with SWB among people with T2DM, implying that resilience can be an important factor contributing to improved SWB. This finding can be interpreted as showing that SWB may benefit from improved social support, resilience and DRSCA. Alternatively, SWB may be a positive stimulus to motivate patients to improve DRSCA.

\section{Limitations}

This study provided a useful step in guiding future research aimed at promoting DRSCA and positive health. However, some limitations should be considered. First, the convenience sampling method may limit the generalisability of the results. Although the sample size was adequate for the analysis, the generalisability is limited by having a hospital sample from one district by none-probability sampling method. However, the impact is not assumed to be substantial because of the wide range of ages and approximately gender balance in this study. Random sampling from different districts in China would allow generalisation of the results to a larger population in the future.

Second, the cross-sectional design allows analysis of many variables at the same time but cannot determine causal relationships. We have taken a first step by conducting a cross-sectional study to determine whether an association exists between DRSCA and positive health, which is the premise of causative relationships but not sufficient for establishing a causal effect. ${ }^{69}$ The design of cross-sectional study had no control group and cannot determine causal relationships. Therefore, its validity is low compared with experimental study. ${ }^{70}$ In the future, studies using an intervention design are needed to further evaluate the causal association of DRSCA and positive health.

Third, only a small percentage of variance of dependent variables was explained. More exploration is needed, including other factors associated with positive health consisting of resilience, SWB and DRQoL. Fourth, the clinical characteristics of the study population including haemoglobin $\mathrm{A} 1 \mathrm{C}(\mathrm{HbA1C})$, lipid profiles, treatment and neuropathy should have been included. We considered that previous studies have provided evidence of the association of DRSCA with HbA1C and lipid profiles, ${ }^{71}$ and only attempted to understand the association of DRSCA with positive health, which is far less known. ${ }^{19}$ Thus, these factors were not included but they can provide more confounders and information. Among the participants with type 2 diabetes followed up at one Affiliated Hospital of Nantong University in another observational study, ${ }^{72}$ average $\mathrm{HbA1C}(\%)$ was 8.7 , triglyceride $(\mathrm{mmol} / \mathrm{L})$ was 1.71 , total cholesterol $(\mathrm{mmol} / \mathrm{L})$ was 4.83 , high density lipoprotein cholesterol (mmol/L) was 1.07 and low density lipoprotein cholesterol $(\mathrm{mmol} / \mathrm{L})$ was 2.67 for those without diabetic peripheral neuropathy (DPN). 
The values were $9.5,1.86,4.93,1.06$ and 2.60 , respectively, for those with DPN. ${ }^{72}$ In this study, the foot care status was surveyed; in future studies, it would be helpful to include neuropathy in order to better understand the outcome of foot care and treatment.

Finally, although self-report measures can provide actionable information as one of the most feasible data collecting methods, ${ }^{73}$ the self-report survey is subject to response bias. ${ }^{74}$ In future research, considering objective measures is suggested. Based on the guideline, Cronbach's alpha coefficients for the scales ranging from 0.64 to 0.94 were considered acceptable. ${ }^{75}$ Another consideration was that the Cronbach's alpha of DRSCA and social support scales was slightly above the low boundary of the range. The internal consistency of DRSCA and social support scales needs further testing in diversified population.

\section{CONCLUSION}

The result about the association between DRSCA and positive health consisting of resilience, SWB and DRQoL may guide intervention development but further research is needed to verify the cause-effect relationship. The results indicate that improving DRSCA, social support and resilience may benefit programmes aimed at enhancing of SWB, which is one component of positive health. The findings may help support patients' motivation to increase the frequency of DRSCA for enhancing SWB. The results also indicate that SWB can be included as an outcome in self-care intervention programmes.

Acknowledgements The authors acknowledge the support of Ai-Juan Wu and Li-Hua Zhao in data collection in the hospitals. We also acknowledge the participants in the study and the research assistants, nurses and doctors for helpful support in data collection. We also thank Anna Vuolteenaho for language revision.

Contributors F-FZ, RS and HL-K designed the study. F-FZ managed the data collection. F-FZ and JK participated in the data analysis. F-FZ drafted the manuscript. F-FZ, RS, HL-K and MS provided important writing and revision of the manuscript.

Funding The work was supported by Nantong City Technology Bureau (BK2014011) and CIMO fellowship from Finland.

Competing interests None declared.

Patient consent for publication Not required

Ethics approval The study protocol was approved by the Ethics Committee of Affiliated Hospital of Nantong University. Informed consent was obtained from participants.

Provenance and peer review Not commissioned; externally peer reviewed.

Data sharing statement No additional data are available.

Open access This is an open access article distributed in accordance with the Creative Commons Attribution Non Commercial (CC BY-NC 4.0) license, which permits others to distribute, remix, adapt, build upon this work non-commercially, and license their derivative works on different terms, provided the original work is properly cited, appropriate credit is given, any changes made indicated, and the use is non-commercial. See: http://creativecommons.org/licenses/by-nc/4.0/.

\section{REFERENCES}

1. Bradley C, Speight J. Patient perceptions of diabetes and diabetes therapy: assessing quality of life. Diabetes Metab Res Rev 2002;18(Suppl 3):S64-9.
2. Donald M, Dower J, Coll JR, et al. Mental health issues decrease diabetes-specific quality of life independent of glycaemic control and complications: findings from Australia's living with diabetes cohort study. Health Qual Life Outcomes 2013;11:170-7525-11-170.

3. IDF Diabetes Atlas 8th edition. 2017. Available: http://www. diabetesatlas.org/resources/2017-atlas.html[Accessed 9 Apr 2018]

4. Chen W, Lou J, Zhu J. Health care utilization and direct economic burden of diabetes patients under one urban health insurance scheme of china. Value Health 2014;17:A253.

5. Toobert DJ, Hampson SE, Glasgow RE. The summary of diabetes self-care activities measure: results from 7 studies and a revised scale. Diabetes Care 2000;23:943-50.

6. Schmitt A, Reimer A, Hermanns N, et al. Assessing Diabetes SelfManagement with the Diabetes Self-Management Questionnaire (DSMQ) Can Help Analyse Behavioural Problems Related to Reduced Glycaemic Control. PLoS One 2016;11:e0150774.

7. Anderson RM, Funnell MM, Butler PM, et al. Patient empowerment. Results of a randomized controlled trial. Diabetes Care 1995;18:943-9

8. Pamungkas RA, Chamroonsawasdi $K$, Vatanasomboon P. A Systematic Review: Family Support Integrated with Diabetes SelfManagement among Uncontrolled Type II Diabetes Mellitus Patients. Behav Sci 2017;7.

9. Fu C, Ji L, Wang W, et al. Frequency of glycated hemoglobin monitoring was inversely associated with glycemic control of patients with Type 2 diabetes mellitus. J Endocrinol Invest 2012;35:269-73.

10. Yang $L$, Wu Q, Hao Y, et al. Self-management behavior among patients with diabetic retinopathy in the community: a structural equation model. Qual Life Res 2017;26:359-66.

11. Yang S, Hsue C, Lou Q. Does patient empowerment predict self-care behavior and glycosylated hemoglobin in chinese patients with type 2 diabetes? Diabetes Technol Ther 2015;17:343-8

12. Simon-Tuval T, Shmueli A, Harman-Boehm I. Adherence to Self-Care Behaviors among Patients with Type 2 Diabetes-The Role of Risk Preferences. Value Health 2016;19:844-51.

13. Blackburn DF, Swidrovich J, Lemstra M. Non-adherence in type 2 diabetes: practical considerations for interpreting the literature. Patient Prefer Adherence 2013;7:183-9.

14. Linmans JJ, Knottnerus JA, Spigt M. How motivated are patients with type 2 diabetes to change their lifestyle? A survey among patients and healthcare professionals. Prim Care Diabetes 2015;9:439-45

15. Choi S, Song M, Chang SJ, et al. Strategies for enhancing information, motivation, and skills for self-management behavior changes: a qualitative study of diabetes care for older adults in Korea. Patient Prefer Adherence 2014;8:219-26.

16. Schulman-Green D, Jaser SS, Park C, et al. A metasynthesis of factors affecting self-management of chronic illness. J Adv Nurs 2016;72:1469-89.

17. Rosenstock IM. Historical origins of the health belief model. Health Educ Behav 1974;2:328-35.

18. Luo X, Liu T, Yuan X, et al. Factors Influencing Self-Management in Chinese Adults with Type 2 Diabetes: A Systematic Review and Meta-Analysis. Int J Environ Res Public Health 2015;12:11304-27.

19. Prendergast KB, Schofield GM, Mackay LM. Associations between lifestyle behaviours and optimal wellbeing in a diverse sample of New Zealand adults. BMC Public Health 2016;16:62.

20. Kvaavik E, Batty GD, Ursin G, et al. Influence of individual and combined health behaviors on total and cause-specific mortality in men and women: the United Kingdom health and lifestyle survey. Arch Intern Med 2010;170:711-8.

21. Seeman J. Toward a model of positive health. Am Psychol 1989;44:1099-109.

22. Locker D, Gibson B. The concept of positive health: a review and commentary on its application in oral health research. Community Dent Oral Epidemiol 2006;34:161-73.

23. Cloninger CR, Salloum IM, Mezzich JE. The dynamic origins of positive health and wellbeing. Int J Pers Cent Med 2012;2:179.

24. Seligman MEP. Positive health. Applied psychology 2008;28.

25. Connor KM, Davidson JR. Development of a new resilience scale: the Connor-Davidson Resilience Scale (CD-RISC). Depress Anxiety 2003;18:76-82.

26. Kumpfer KL. Factors and processes contributing to resilience: The resilience framework. In: Glantz MD, Johnson JL, eds. Resilience and development: Positive life adaptations. New York: Kluwer Academic/ Plenum Publishers, 1999:179-224.

27. Cal SF, de Sá LR, Glustak ME, et al. Resilience in chronic diseases: A systematic review. Cogent Psychol 2015;2:1024928.

28. Sun N, Lou P, Shang Y, et al. Prevalence and determinants of depressive and anxiety symptoms in adults with type 2 diabetes in China: a cross-sectional study. BMJ Open 2016;6:e012540. 
29. Ezeamama AE, Elkins J, Simpson C, et al. Indicators of resilience and healthcare outcomes: findings from the 2010 health and retirement survey. Qual Life Res 2016;25:1007-15.

30. Saletnik L. Building Personal Resilience. Aorn J 2018;107:175-8.

31. Olsson CA, Bond L, Burns JM, et al. Adolescent resilience: a concept analysis. J Adolesc 2003;26:1-11.

32. Lloyd C, Campion DP. Occupational stress and the importance of self-care and resilience: focus on veterinary nursing. Ir Vet $J$ 2017;70.

33. Diener E. Subjective well-being. The science of happiness and a proposal for a national index. Am Psychol 2000;55:34-43.

34. Ryan RM, Deci EL. On happiness and human potentials: a review of research on hedonic and eudaimonic well-being. Annu Rev Psychol 2001;52:141-66.

35. World Health Organization. The European health report 2012: chartering the way to well-being. Available: http://www.euro.who.int/ en/publications/abstracts/european-health-report-2012-charting-theway-to-well-being-the.-executive-summary

36. Cubí-Mollá P, de Vries J, Devlin N. A study of the relationship between health and subjective well-being in Parkinson's disease patients. Value Health 2014;17:372-9.

37. Hansson A, HilleråS P, Forsell Y. What Kind of Self-Care Strategies Do People Report Using and is There an Association with WellBeing? Soc Indicators Res 2005;73:133-9.

38. El Achhab Y, Nejjari C, Chikri M, et al. Disease-specific health-related quality of life instruments among adults diabetic: A systematic review. Diabetes Res Clin Pract 2008;80:171-84.

39. Wang RH, Wu LC, Hsu HY. A path model of health-related quality of life in type 2 diabetic patients: a cross-sectional study in Taiwan. $J$ Adv Nurs 2011;67:2658-67.

40. Chen CY, Lo FS, Chen BH, et al. Pathways of emotional autonomy, self-care behaviors, and depressive symptoms on health adaptation in adolescents with type 1 diabetes. Nurs Outlook 2017;65:68-76.

41. Yoon PW, Scheuner MT, Peterson-Oehlke KL, et al. Can family history be used as a tool for public health and preventive medicine? Genet Med 2002;4:304-10.

42. Mayberry LS, Osborn CY. Family support, medication adherence, and glycemic control among adults with type 2 diabetes. Diabetes Care 2012;35:1239-45.

43. Lehti J. Theory of psychological adaptive modes. Med Hypotheses 2016;90:66-73.

44. Zhao FF, Suhonen R, Katajisto J, et al. The association of diabetesrelated self-care activities with perceived stress, anxiety, and fatigue: a cross-sectional study. Patient Prefer Adherence 2018;12:1677-86.

45. Satija A, Yu E, Willett WC, et al. Objective measures are complementary to, rather than a replacement for, self-reported methods. Int J Obes 2015;39:1179.

46. Xiao SY. The theoretical foundation of the social support rating scale and applications in research. J Clini Psycho Med 1994;4:98-100.

47. Dai W, Chen L, Tan H, et al. Association between social support and recovery from post-traumatic stress disorder after flood: a 13-14 year follow-up study in Hunan, China. BMC Public Health 2016;16:194.

48. Yin Xu, Savage C, Toobert D, et al. Adaptation and testing of instruments to measure diabetes self-management in people with type 2 diabetes in mainland China. J Transcult Nurs 2008;19:234-42.

49. Campbell-Sills L, Stein MB. Psychometric analysis and refinement of the Connor-davidson Resilience Scale (CD-RISC): Validation of a 10-item measure of resilience. J Trauma Stress 2007;20:1019-28.

50. Bech P, Gudex C, Johansen KS. The WHO (Ten) Well-Being Index: validation in diabetes. Psychother Psychosom 1996;65:183-90.

51. Bech $\mathrm{P}$, Olsen RL, Kjoller M, et al. Measuring well-being rather than the absence of distress symptoms: a comparison of the SF-36 Mental Health subscale and the WHO- Five Well-Being Scale. Int $J$ Meth Psychiatr Res 2003;12:85-91.

52. Topp CW, Østergaard SD, Søndergaard S, et al. The WHO-5 Well-Being Index: a systematic review of the literature. Psychother Psychosom 2015;84:167-76.

53. Kong D, Ding $Y, Z u o X$, et al. Adaptation of the Audit of DiabetesDependent Quality of Life questionnaire to people with diabetes in China. Diabetes Res Clin Pract 2011;94:45-52.
54. Faul F, Erdfelder E, Buchner A, et al. G*Power Version 3.1.7 [computer software]. Vol. Available: http://www.gpower.hhu.de/

55. Van den Broeck J, Cunningham SA, Eeckels R, et al. Data cleaning: detecting, diagnosing, and editing data abnormalities. PLoS Med 2005;2:e267.

56. Imperatore G, Boyle JP, Thompson TJ, et al. Projections of type 1 and type 2 diabetes burden in the U.S. population aged $<20$ years through 2050: dynamic modeling of incidence, mortality, and population growth. Diabetes Care 2012;35:2515-20.

57. "Causes of Diabetes" National Institute of Diabetes and Digestive and Kidney Diseases. 2014. https://www.niddk.nih.gov/healthinformation/diabetes/overview/symptoms-causes [Accessed $10 \mathrm{Feb}$ 2016].

58. Böell JE, Silva DM, Hegadoren KM. Sociodemographic factors and health conditions associated with the resilience of people with chronic diseases: a cross sectional study. Rev Lat Am Enfermagem 2016;24:e2786

59. Pichon-Riviere A, Irazola V, Beratarrechea A, et al. Quality of life in type 2 diabetes mellitus patients requiring insulin treatment in Buenos Aires, Argentina: a cross-sectional study. Int $J$ Health Policy Manag 2015;4:475-80.

60. Ostini R, Dower J, Donald M. The Audit of Diabetes-Dependent Quality of Life 19 (ADDQoL): feasibility, reliability and validity in a population-based sample of Australian adults. Qual Life Res 2012;21:1471-7.

61. de Wit M, Pouwer F, Gemke RJ, et al. Validation of the WHO-5 Well-Being Index in adolescents with type 1 diabetes. Diabetes Care 2007;30:2003-6.

62. Goodwin LD, Leech NL. Understanding Correlation: Factors That Affect the Size of r. J Exp Educ 2006;74:251-66.

63. Khazrai YM, Defeudis G, Pozzilli P. Effect of diet on type 2 diabetes mellitus: a review. Diabetes Metab Res Rev 2014;30(Suppl 1):24-33.

64. Conn VS. Helping patients help themselves: chronic disease selfmanagement interventions. West J Nurs Res 2011;33:159-60.

65. Khuwaja AK, Lalani S, Dhanani R, et al. Anxiety and depression among outpatients with type 2 diabetes: A multi-centre study of prevalence and associated factors. Diabetol Metab Syndr 2010;2:72.

66. Beck AT, Haigh EA. Advances in cognitive theory and therapy: the generic cognitive model. Annu Rev Clin Psychol 2014;10:1-24.

67. Siedlecki KL, Salthouse TA, Oishi S, et al. The Relationship Between Social Support and Subjective Well-Being Across Age. Soc Indic Res 2014;117:561-76.

68. Karlsen B, Bru E. The relationship between diabetes-related distress and clinical variables and perceived support among adults with type 2 diabetes: a prospective study. Int J Nurs Stud 2014;51:438-47.

69. Causation and Experimental Design. 2006:P106-35. https://uk. sagepub.com/sites/default/files/upm- binaries/23639_Chapter 5 _Causation_and_Experimental_Design.pdf. 05-Chambliss.qxd [Accessed 12 Jan 2018]

70. Carlson MD, Morrison RS. Study design, precision, and validity in observational studies. J Palliat Med 2009;12:77-82.

71. Rosal MC, Ockene IS, Restrepo A, et al. Randomized trial of a literacy-sensitive, culturally tailored diabetes self-management intervention for low-income latinos: latinos en control. Diabetes Care 2011:34:838-44

72. Su JB, Zhao LH, Zhang XL, et al. HbA1c variability and diabetic peripheral neuropathy in type 2 diabetic patients. Cardiovasc Diabetol 2018;17:47.

73. Stirratt MJ, Dunbar-Jacob J, Crane HM, et al. Self-report measures of medication adherence behavior: recommendations on optimal use. Trans/ Behav Med 2015;5:470-82.

74. Johnson J, Louch G, Dunning A, et al. Burnout mediates the association between depression and patient safety perceptions: a cross-sectional study in hospital nurses. J Adv Nurs 2017;73:1667-80.

75. AGREE Collaboration. Development and validation of an international appraisal instrument for assessing the quality of clinical practice guidelines: the AGREE project. Qual Saf Health Care 2003;12:18-23. 